01

\title{
Внутридоплеровские резонансы, обусловленные фотоионизацией атомов в тонких газовых ячейках
}

\author{
(C) А.Ч. Измайлов \\ Институт фризики НАН Азербайджана, \\ AZ-1143 Баку, Азербайджан \\ e-mail: azizm57@rambler.ru
}

Поступила в редакцию 02.10.2021 г.

В окончательной редакции 17.11.2021 г.

Принята к публикации 19.11.2021 г.

По аналогии с хорошо апробированным методом спектроскопии высокого разрешения в тонких газовых ячейках для процессов оптической накачки атомов показана возможность регистрации узких внутридоплеровских оптических резонансов, обусловленных непосредственно прямой фотоионизацией атомов (или молекул) в подобных ячейках. Структура установленных нетривиальных резонансов существенно зависит от вероятности фотоионизации атомов и размеров ячейки, внутренняя толщина которой во много раз меньше ее диаметра. Особый интерес представляет уширение рассматриваемых внутридоплеровских резонансов, которое определяется непосредственно сечением фотоионизации атомов и интенсивностью излучения, вызывающего ионизацию. При определенных условиях такое фотоионизационное уширение может быть измерено на эксперименте с высокой точностью, в результате чего возможно получение новой важной информации об ионизационных процессах в атомах и молекулах.

Ключевые слова: внутридоплеровские резонансы, фотоионизация атомов, тонкая газовая ячейка, пролетная релаксация атомов.

DOI: $10.21883 /$ OS.2022.03.52161.2785-21

\section{Введение}

Исследование процессов фотоионизации в разреженных газовых средах имеет важное значение для атомной и молекулярной спектроскопии, физики плазмы и астрофизики [1-4]. Прямая однофотонная ионизация из основного атомного (молекулярного) квантового состояния может осуществляться ультрафиолетовым или вакуумным ультрафиолетовым излучением, которое, в частности, генерируется синхротронами и некоторыми газоразрядными лампами, а также присутствует в солнечной радиации [1-3]. В то же время многофотонная ионизация атомов производится достаточно интенсивным лазерным излучением видимого или инфракрасного диапазонов [4].

Эффективность прямой фотоионизации индивидуальных атомов (или молекул) определяется не только интенсивностью ионизирующего излучения, но и временем его воздействия на частицу. Поэтому в случае достаточно разреженной газовой среды в ячейке процесс фотоионизации будет существенно зависеть от характерных времен пролета атомов (молекул) между стенками такой ячейки. Подобная ситуация характерна для оптической накачки атомов, при которой происходит светоиндуцированное перераспределение населенностей подуровней их основного квантового терма [5]. В работах [6-9] были предложены новые методы внутридоплеровской лазерной спектроскопии, основанные на специфике стационарной оптической накачки атомов в течение их пролета между стенками тонкой газовой ячейки, у которой характерный поперечный размер $D$ во много раз больше, чем ее внутренняя толщина $L$. Впоследствии данные методы были успешно реализованы на экспериментах для прецизионного спектрального анализа атомов [10-15] на основе серии тонких ячеек (с различной внутренней толщиной вплоть до $10 \mu \mathrm{m})$, содержащих пары цезия [10-14] или рубидия [15]. Работа [16] представляет обзор этих методов, достижений и возможностей спектроскопии сверхвысокого разрешения в таких ячейках. В частности, был разработан и апробирован метод внутридоплеровской спектроскопии, в котором оптическая накачка атомов может осуществляться даже широкополосным излучением во всем объеме тонкой ячейки, а их зондирование производится пробным монохроматическим световым пучком [7,9,12-16].

В настоящей работе показано, что подобный метод спектроскопии может быть использован также для анализа фотоионизации атомов (молекул) разреженного газа. Действительно, рассмотрим схему эксперимента на рис. 1, где по всему объему тонкой ячейки осуществляется прямая фотоионизация атомов из их основного квантового состояния $a$ в состояние континуума $i$ (рис. 2). В рассматриваемой ситуации достаточно разреженного газа уменьшение количества атомов на уровне $a$ изза процесса ионизации определяется временем их пролета между стенками ячейки, на которых происходит релаксация атомных частиц к равновесному состоянию. В нашем случае такая релаксация обусловлена, главным образом, столкновениями атомов с торцевыми (плоскопараллельными) стенками тонкой ячейки (рис. 1). Поэтому процессы прямой фотоионизации и пролетной релаксации атомов в подобной ячейке приводят к узкому 


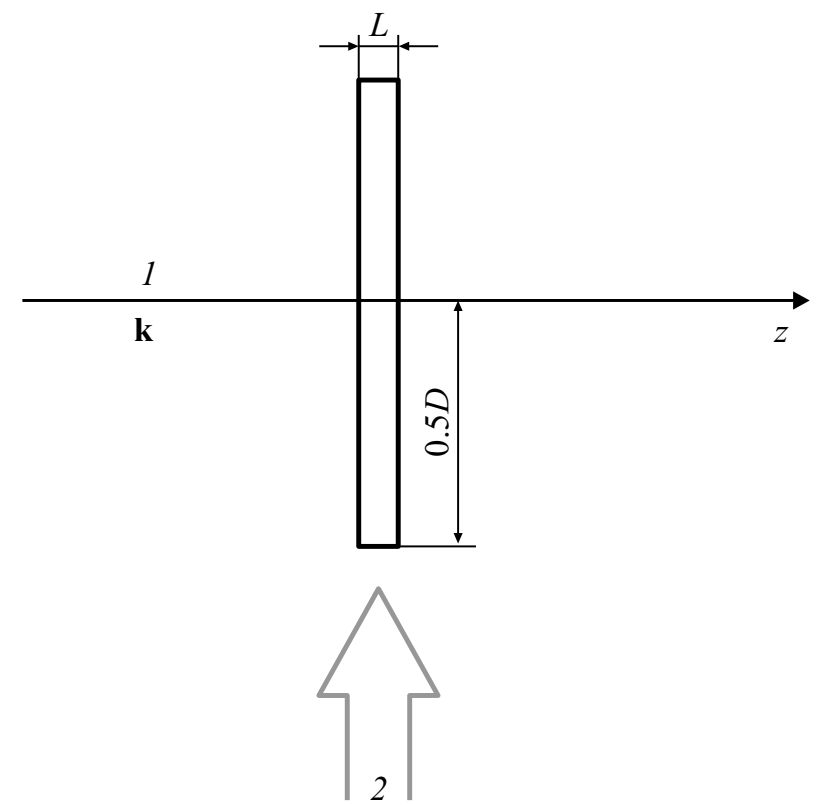

Рис. 1. Схема эксперимента с тонкой газовой ячейкой $\mathrm{c}$ диаметром $D$ и внутренней толщиной $L \ll D$, где $1-$ пробный монохроматический световой пучок, 2 - ионизирующее излучение.

провалу в распределении электронейтральных атомов по проекции $v_{z}$ их скорости вдоль оси $z$ ячейки в окрестности значения $v_{z}=0$. Таким образом, могут возникнуть внутридоплеровские резонансы в спектре поглощения пробной монохроматической световой волны с волновым вектором $\mathbf{k}$, направленным вдоль оси $z$ (рис. 1), на центральных частотах оптических переходов из основного квантового состояния $a$ на какие-либо возбужденные уровни электронейтральных атомов (рис. 2). В настоящей работе показано, что структура этих детектируемых узких спектральных резонансов будет существенно зависеть от вероятности процесса фотоионизации. В результате открываются новые возможности для определения сечения фотоионизации атомов (молекул) и интенсивности ионизирующего излучения методами спектроскопии сверхвысокого разрешения.

\section{Основные соотношения}

Рассмотрим прямую фотоионизацию атомов (или молекул) газовой среды стационарным излучением, однородным по всему объему тонкой цилиндрической газовой ячейки (рис. 1), которая сделана из материала, прозрачного как для ионизирующего, так и для пробного излучений. Предполагается, что в данном достаточно разреженном газовом слое можно пренебречь межатомным взаимодействием. Слабая пробная монохроматическая световая волна с волновым вектором $\mathbf{k}$ и сравнительно малым диаметром $d \ll D$ распространяется через центр ячейки вдоль оси $z$ (рис. 1). Частота $\omega$ данной волны сканируется в окрестности центра $\omega_{0}$ некоторого оптического перехода $a \rightarrow b$ с основного квантового уровня $a$ на возбужденный уровен $b$ электронейтральных атомов (рис. 2). При рассматриваемых условиях имеет место следующее балансное уравнение для населенности $\rho_{a}(\mathbf{r}, \mathbf{v})$ атомов в основном состоянии $a$ со скоростью $\mathbf{v}$ и координатным вектором $\mathbf{r}$ :

$$
\mathbf{v} \frac{\partial \rho_{a}}{\partial \mathbf{r}}=-\xi \rho_{a},
$$

где $\xi-$ вероятность (в единицу времени) прямой фотоионизации атомов из состояния $a$ (рис. 2), которая определяется интенсивностью ионизирующего излучения и сечением фотоионизации. Уравнение (1) должно быть дополнено граничными условиями, зависящими от особенностей столкновений со стенками ячейки электронейтральных атомов и образующихся фотоионов. Как и в расчетах [6-16], выполненных для процессов оптической накачки в тонких газовых ячейках, будем полагать, что в результате таких столкновений устанавливается равновесное распределение как по скоростям атомов, так и по населенностям их квантовых уровней. Важно отметить, что рассматривается не слишком высокая интенсивность ионизации, когда количество возникающих фотоионов и фотоэлектронов во много раз меньше числа атомов в ячейке. Вследствие процесса перезарядки ионов на поверхности [17] будем считать, что после нескольких столкновений фотоиона со стенками ячейки происходит его нейтрализация, т.е. восстановление атома. Пренебрегаем также влиянием возникающих фотоэлектронов

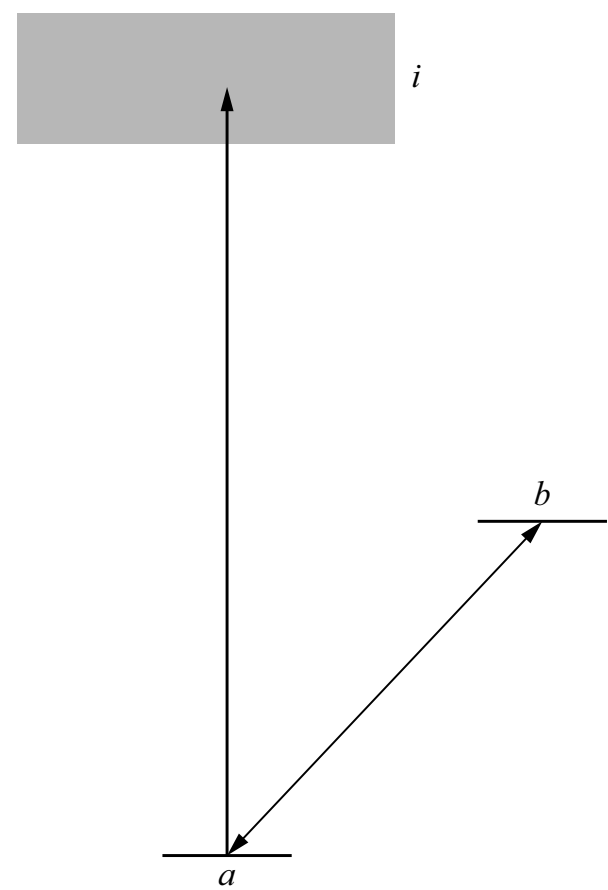

Рис. 2. Схема рассматриваемых квантовых уровней и переходов: $a \rightarrow i-$ прямая фотоионизация атомов из основного терма $a$ в состояние ионизационного континуума $i, a \rightarrow b-$ оптический переход из состояния $a$ в возбужденное состояние $b$, зондируемый пробным монохроматическим световым пучком. 
на распределение электронейтральных атомов газового слоя. Тогда из уравнения (1) получаем следующее выражение для населенности $\rho_{a}(\mathbf{r}, \mathbf{v})$ основного уровня $a$ атомов в области пробного светового пучка, сконценрированного около центральной оси ячейки $z$ (рис. 1) в интервале $-0.5 L \leq z \leq 0.5 L$ :

$$
\begin{aligned}
& \rho_{a}\left(z, v_{z}, v_{r}\right)=n_{a} F_{l}\left(v_{z}\right) F_{r}\left(v_{r}\right) \\
& \quad \times\left\{\left[\exp \left(-\xi \frac{(z+0.5 L)}{v_{z}}\right) \eta\left(\frac{D v_{z}}{2 v_{r}}-z-0.5 L\right)\right.\right. \\
& \left.\quad+\exp \left(-\xi \frac{D}{2 v_{r}}\right) \eta\left(z+0.5 L-\frac{D v_{z}}{2 v_{r}}\right)\right] \eta\left(v_{z}\right) \\
& \quad+\left[\exp \left(\frac{-\xi(z-0.5 L)}{v_{z}}\right) \eta\left(z-0.5 L-\frac{D v_{z}}{2 v_{r}}\right)\right. \\
& \left.\left.\quad+\exp \left(-\xi \frac{D}{2 v_{r}}\right) \eta\left(\frac{D v_{z}}{2 v_{r}}-z+0.5 L\right)\right] \eta\left(-v_{z}\right)\right\}
\end{aligned}
$$

где $\eta(x)$ - ступенчатая функция $(\eta(x)=1$, если $x \geq 0$ и $\eta(x)=0$, когда $x<0), n_{a}$ - равновесная плотность атомов на уровне $a$ с распределениями Максвелла $F_{l}\left(v_{z}\right)$ и $F_{r}\left(v_{r}\right)$ соответственно по продольной $v_{z}$ и радиальной $v_{r}$ компонентам атомной скорости $\mathbf{v}$ :

$$
\begin{gathered}
F_{l}\left(v_{z}\right)=\pi^{-0.5} u^{-1} \exp \left(-v_{z}^{2} u^{-2}\right), \\
F_{r}\left(v_{r}\right)=2 v_{r} u^{-2} \exp \left(-v_{r}^{2} u^{-2}\right),
\end{gathered}
$$

с наиболее вероятной скоростью $u$ атомов в газе. Из выражения (2) получаем следующее распределение по проекции скорости $v_{z}$ для усредненной населенности $N_{a}\left(v_{z}\right)$ атомов, сосредоточенных около центральной оси ячейки $z$ (рис. 1):

$$
N_{a}\left(v_{z}\right)=L^{-1} \int_{-0.5 L}^{0.5 L}\left[\int_{0}^{\infty} \rho_{a}\left(z, v_{z}, v_{r}\right) d v_{r}\right] d z .
$$

Далее, как и в работах [6-16] со стационарной оптической накачкой, будем полагать, что толщина ячейки $L \gg 1 \mu \mathrm{m}$, т.е. во много раз превосходит длину волны оптического излучения. Тогда линейный коэффициент поглощения $G$ слабой пробной оптической волны на переходе $a \rightarrow b$ (рис. 2) в рассматриваемой газовой ячейке имеет вид

$$
G(\delta)=G_{0} \frac{\left\{\int_{-\infty}^{\infty} N_{a}\left(v_{z}\right)\left[\gamma^{2}+\left(\delta-k v_{z}\right)^{2}\right]^{-1} d v_{z}\right\}}{\left\{\int_{-\infty}^{\infty} n_{a} F_{l}\left(v_{z}\right)\left[\gamma^{2}+\left(k v_{z}\right)^{2}\right]^{-1} d v_{z}\right\}}
$$

где населенность $N_{a}\left(v_{z}\right)$ определяется формулой (4), $k=|\mathbf{k}|=\omega / c, \delta=\left(\omega-\omega_{0}\right)$ - отстройка сканируемой частоты $\omega$ от центра $\omega_{0}$ оптического перехода $a \rightarrow b$ (рис. 2) с естественной полушириной $\gamma$ и доплеровским уширением $k u \gg \gamma$ спектральной линии, множитель $G_{0}$ - коэффициент поглощения пробной волны на данном переходе $a \rightarrow b$ в отсутствие ионизирующего излучения и при нулевой отстройке $\delta=0$.

\section{Обсуждение результатов}

На рис. 3, $a$ представлено распределение $N_{a}\left(v_{z}\right)$ (4) населенности атомов на основном уровне $a$ по атомной проекции скорости $v_{z}$ при различных вероятностях фотоионизации $\xi$ и отношений $D / L \gg 1$ размеров тонкой ячейки (рис. 1). Видно, что в зависимости $N_{a}\left(v_{z}\right)$ на фоне сравнительно широкого скоростного распределения с характерной шириной, определяемой наиболее вероятной скоростью атомов $u$, возникает узкий симметричный провал с центром в точке $v_{z}=0$. Данный провал обусловлен спецификой прямой фотоионизации и пролетной релаксации атомов в рассматриваемой ячейке. Его происхождение легко понять в модели плоской одномерной ячейки с отношением размеров $D / L \rightarrow \infty$. Тогда, согласно уравнению (1), эффективная ионизация атомов осуществляется при условии

$$
\xi \frac{L}{\left|v_{z}\right|} \geq 1
$$

т.е. при достаточно большом времени пролета $L /\left|v_{z}\right|$ атомов между плоскопараллельными стенками ячейки. Из (6) непосредственно следует резкое усиление процесса ионизации атомов при $\left|v_{z}\right| \rightarrow 0$, в результате чего и возникает узкий центральный провал в распределении $N_{a}\left(v_{z}\right)$ на рис. $3, a$.

В случае газовой ячейки с реальным поперечным размером $D$ для эффективной фотоионизации атомов наряду с соотношением (6) должно выполняться также следующее условие для их радиальной компоненты скорости $v_{r}$ :

$$
\xi \frac{D}{2\left|v_{r}\right|} \geq 1
$$

Данное условие связано с характерным временем $0.5 D /\left|v_{r}\right|$ пролета атомов в поперечном направлении к центральной области ячейки, зондируемой пробным световым пучком (рис. 1).

Согласно соотношениям (6), (7), рассматриваемый центральный провал в распределении $N_{a}\left(v_{z}\right)$ на рис. $3, a$ увеличивается с ростом вероятности фотоионизации $\xi$ и диаметра $D$ тонкой ячейки (при ее фиксированной внутренней толщине $L$ ).

Количество фотоионизованных атомов в ячейке определяется величиной $\Delta N_{a}\left(v_{z}\right)$ :

$$
\Delta N_{a}\left(v_{z}\right)=n_{a} F_{l}\left(v_{z}\right)-N_{a}\left(v_{z}\right) .
$$

Соответствующие зависимости $\Delta N_{a}\left(v_{z}\right)$ представлены на рис. $3, b$ и непосредственно отражают структуру провала в распределении $N_{a}\left(v_{z}\right)$ на рис. $3, a$. Заметим, что интегральное количество $\int_{-\infty}^{\infty} \Delta N_{a}\left(v_{z}\right) d v_{z}$ образующихся фотоионов и фотоэлектронов в нашем случае во много раз меньше числа электронейтральных атомов.

Узкий центральный провал в скоростном распределении $N_{a}\left(v_{z}\right)$ (рис. 3,a) приводит к возникновению внутридоплеровского резонанса с центром при нулевой частотной отстройке $\delta=0$ в спектральной зависимости коэффициента поглощения $G(\delta)(5)$ пробного 

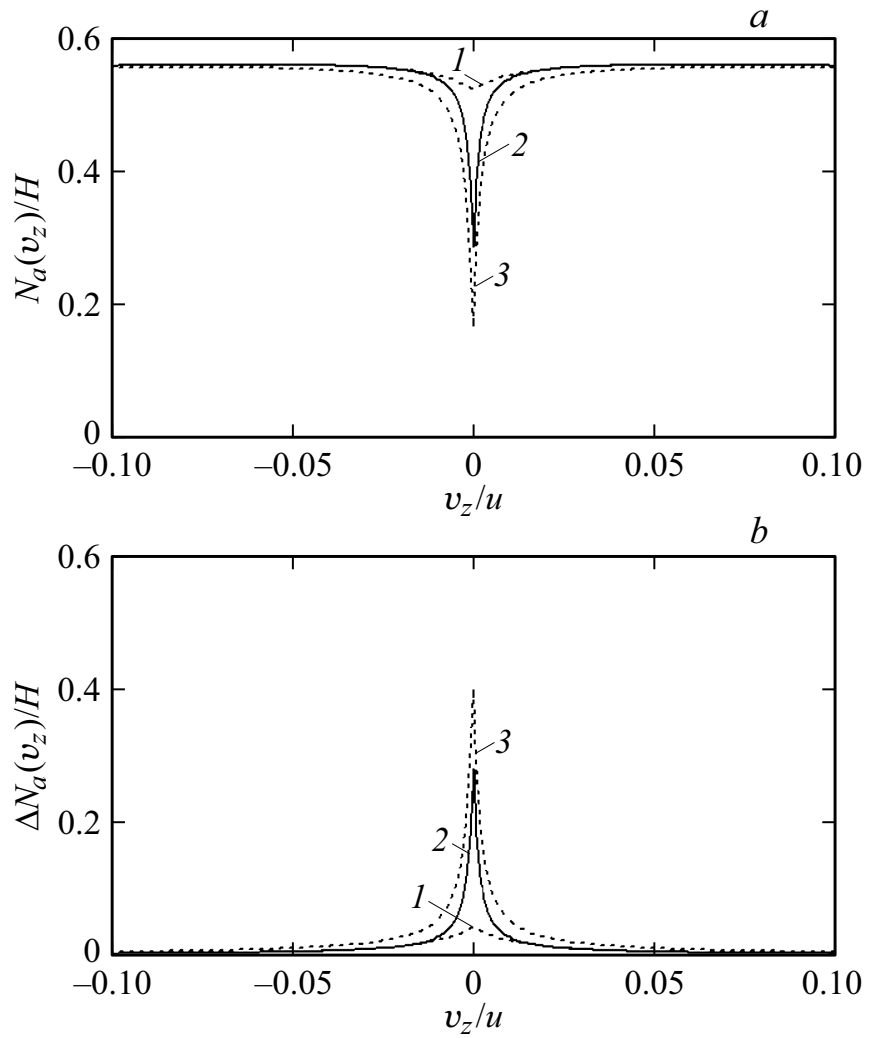

Рис. 3. Распределения $N_{a}\left(v_{z}\right)$ (a) и $\Delta N_{a}\left(v_{z}\right)$ (b) атомов в основном квантовом состоянии $a$ по компоненте атомной скорости $v_{z}$ (в единицах $\left.u\right)$, когда $\xi(L / u)=0.001(1,2)$ и $0.002(3)$, а $(D / L)=100(1)$ и $1000(2,3)$. Данные функции $N_{a}\left(v_{z}\right)$ и $\Delta N_{a}\left(v_{z}\right)$ нормированы на величину $H=n_{a} u^{-1}$.

монохроматического светового пучка. Такие узкие резонансы могут, в частности, наблюдаться как разность между сигналами поглощения пробной волны, которые последовательно регистрируются в присутствии и в отсутствие ионизирующего излучения (рис. 1). Тогда детектируемая часть $\Delta G(\delta)$ коэффициента поглощения получается из выражения $G(\delta)(5)$ заменой $N_{a}\left(v_{z}\right)$ на $\Delta N_{a}\left(v_{z}\right)$ (8). Другой метод регистрации подобных внутридоплеровских резонансов возможен посредством достаточно малой модуляции частоты пробного излучения, когда определяется непосредственно частотная производная $d G(\delta) / d \delta$ коэффициента поглощения $G(\delta)(5)$. Такие спектральные зависимости $\Delta G(\delta)$ и $d G(\delta) / d \delta$ представлены на рис. 4. Так же как и соответствующий центральный провал в скоростном распределении $N_{a}\left(v_{z}\right)$ (рис. 3), рассматриваемые внутридоплеровские структуры на рис. 4 увеличиваются с ростом вероятности фотоионизации $\xi$ и отношения $D / L$ размеров тонкой ячейки (рис. 1). Особый интерес представляет анализ характерных ширин этих внутридоплеровских резонансов, которые могут быть измерены на эксперименте с весьма высокой точностью известными методами оптической спектроскопии [5]. В случае резонанса $\Delta G(\delta)$ (рис. 4, $a$ ) с амплитудой $A_{1}=\Delta G(\delta=0)$ в качестве такой ширины $w_{1}$ будем рассматривать ее значение на полувысоте зависимости $\Delta G(\delta)$. Для внутридоплеровского резонанса дисперсионной формы на рис. $4, b$ его характерную ширину $w_{2}$ будем определять по частотному интервалу между максимумом и минимумом функции $d G(\delta) / d \delta$, a его амплитуду $A_{2}$ - по разности между этими экстремумами.

На рис. 5, $a$ и 6, $a$ представлены зависимости ширин $w_{1}$ и $w_{2}$ исследуемых внутридоплеровских резонансов (рис. 4) от вероятности $\xi$ фотоионизации атомов при различных отношениях $D / L$ размеров тонкой ячейки с фиксированной внутренней толщиной $L$ (рис. 1). Кривые 3 на рис. 5 и 6 соответствуют модели плоской одномерной ячейки, когда $D / L \rightarrow \infty$. При предельно низкой вероятности фотоионизации $\xi(L / u) \rightarrow 0$ ширины, описываемые кривыми 3 , асимптотически приближаются к значениям $w_{10} \approx 2.5 \gamma$ (рис. $5, a$ ) и $w_{20} \approx 1.25 \gamma$ (рис. 6, $a$ ), которые соответствуют естественному уширению рассматриваемых внутридоплеровских резонансов. В случае ячеек с реальными поперечными размерами $D$ (рис. 1) в этих резонансах проявится также остаточное доплеровское уширение порядка величины $(L / D) k u$ (кривые 1 и 2 на рис. 5, $a$ и $6, a)$. Действительно, согласно скоростному распределению $\Delta N_{a}\left(v_{z}\right)$ на рис. $3, b$, совокупность фотоионизованных атомов явля-
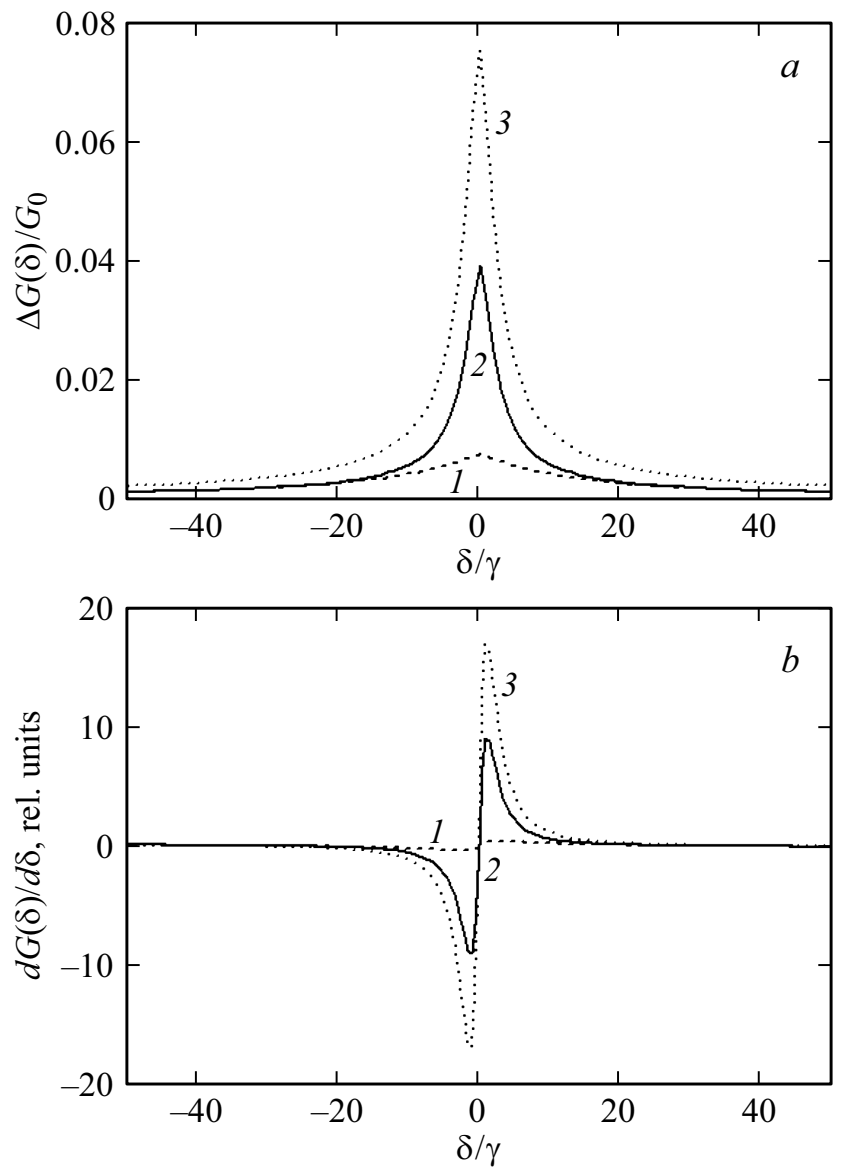

Рис. 4. Внутридоплеровские резонансы $\Delta G(\delta)(a)$ и $\frac{d G(\delta)}{d \delta}(b)$ в зависимости от расстройки частоты $\delta$ (в единицах $\gamma$ ), когда $\gamma=10^{-3} k u, \xi(L / u)=0.0001(1,2)$ и $0.0002(3)$, а $(D / L)=100(1)$ и $1000(2,3)$. 
ется компактным аналогом атомного пучка с характерной расходимостью $\sim(L / D)$. Поэтому с уменьшением отношения $D / L$ происходит рост ширин соответствующих резонансов на рис. 5, $a$ и $6, a$. Ионизация атомов приводит к дополнительному уширению данных резонансов. Начиная с некоторых значений вероятности $\xi$, такое фотоионизационное уширение становится доминирующим, в результате чего асимптотически исчезает различие между ширинами внутридоплеровских резонансов, полученных при различных отношениях размеров ячейки $(D / L) \gg 1$ (кривые 2 и 3 на рис. 5, $a$ и 6, $a$ ). При этом зависимости ширин $w_{1}$ и $w_{2}$ от вероятности фотоионизации $\xi$ носят квазилинейный характер.

Согласно рис. 5, $b$, амплитуда $A_{1}$ внутридоплеровского резонанса $\Delta G(\delta)$ увеличивается с ростом вероятности фотоионизации $\xi$ и диаметра $D$ тонкой ячейки (при ее фиксированной внутренней толщине $L$ ). Это находится в соответствии с зависимостью $\Delta N_{a}\left(v_{z}\right)(8)$, представлен-
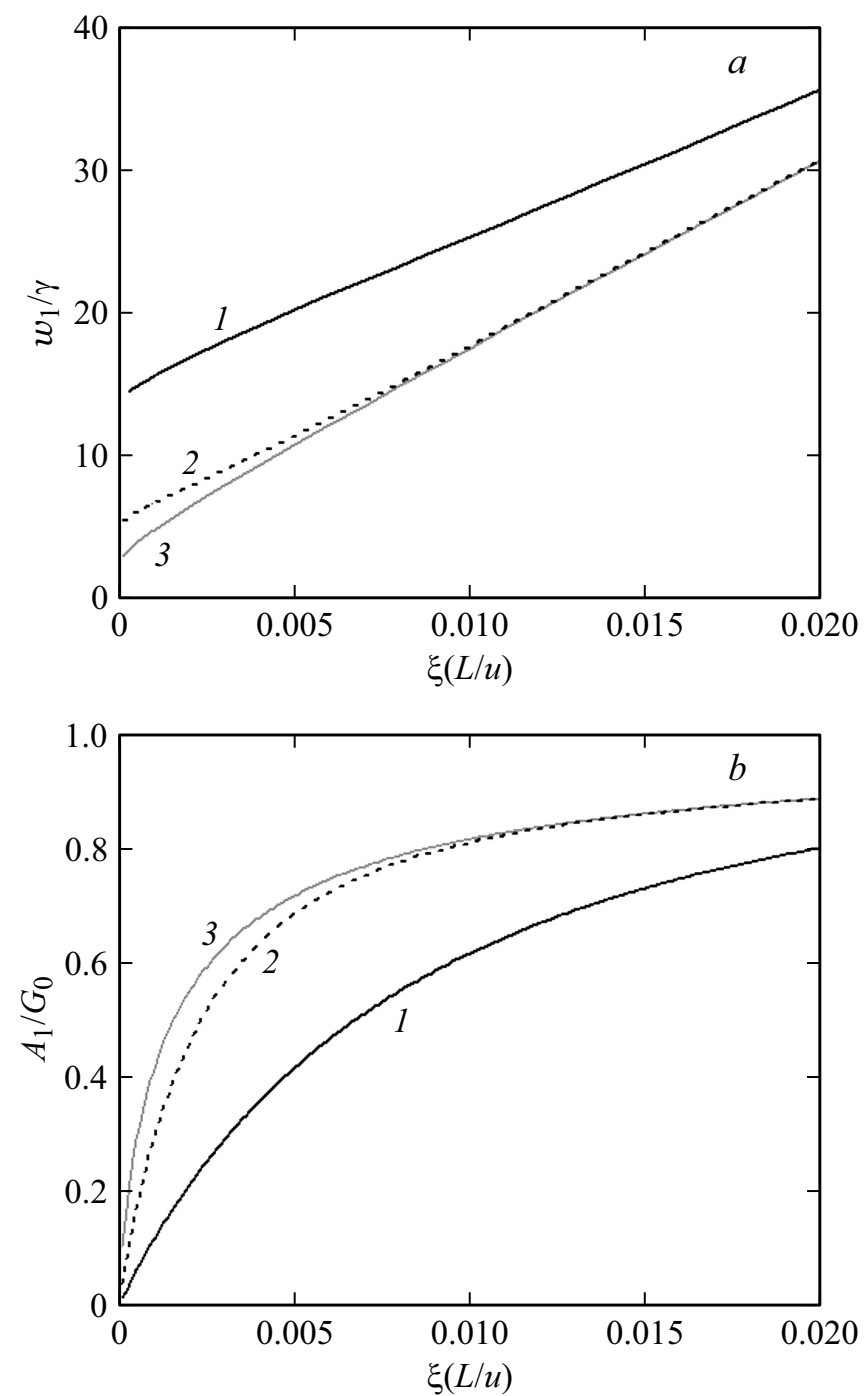

Рис. 5. Зависимости ширины $w_{1}(a)$ и амплитуды $A_{1}(b)$ внутридоплеровского резонанса $\Delta G(\delta)$ от вероятности фотоионизации $\xi$ (в единицах $u / L)$, когда $\gamma=10^{-3} k u,(D / L)=200(1)$, $1000(2)$ и $\infty(3)$.
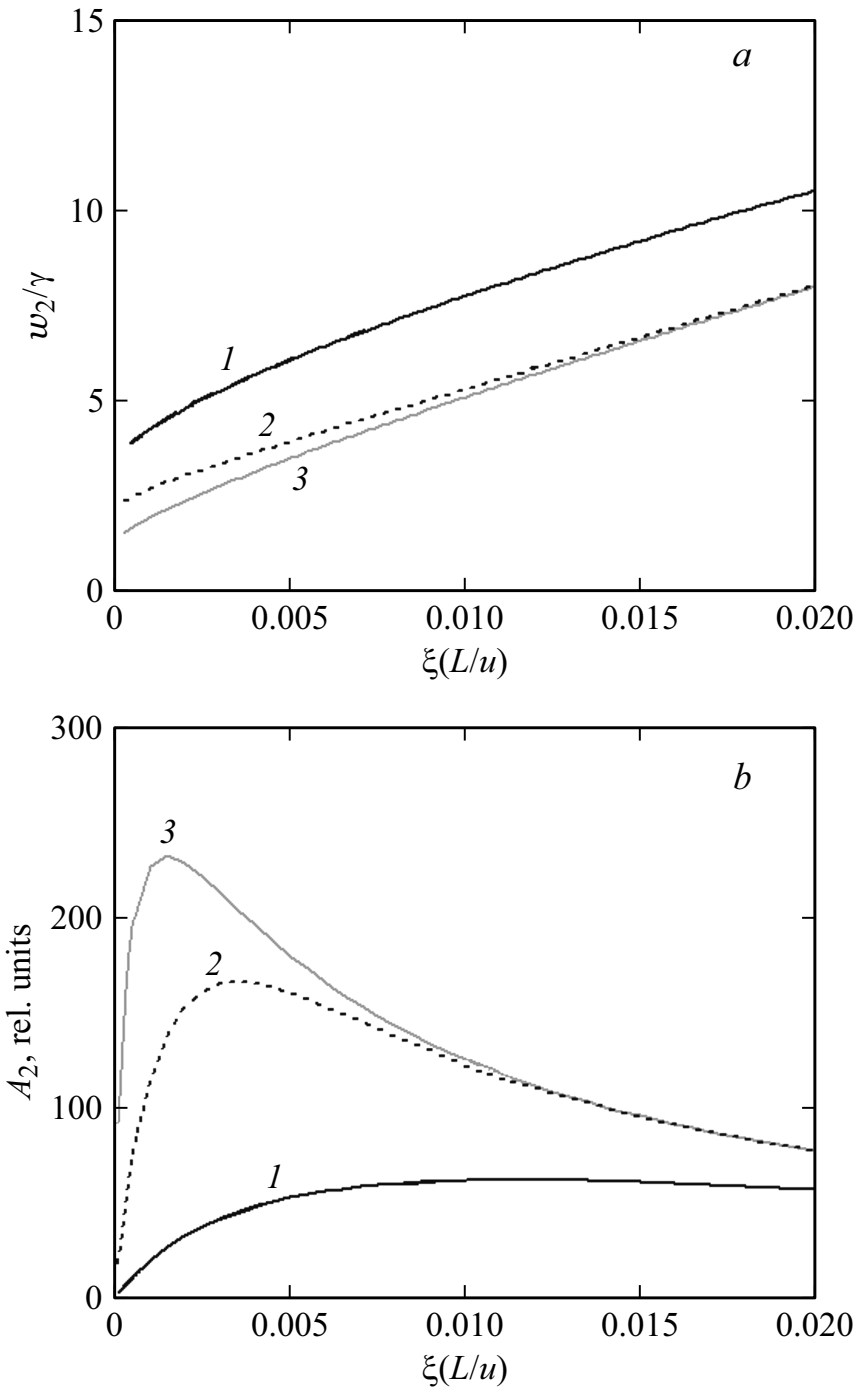

Рис. 6. Зависимости ширины $w_{2}(a)$ и амплитуды $A_{2}(b)$ внутридоплеровского резонанса $d G(\delta) / d \delta$ от вероятности фотоионизации $\xi$ (в единицах $u / L)$, когда $\gamma=10^{-3} k u$, $(D / L)=200(1), 1000(2)$ и $\infty(3)$.

ной на рис. $3, b$. В то же время с ростом вероятности фотоионизации $\xi$ амплитуда $A_{2}$ функции $d G(\delta) / d \delta$ сначала увеличивается до определенного значения, после чего происходит ее монотонное уменьшение (рис. 6, $b$ ). При достаточно малых значениях $\xi$ зависимости амплитуд $A_{1}(\xi)$ и $A_{2}(\xi)$ носят квазилинейный характер (рис. $5, b$ и $6, b)$.

\section{Заключение}

В настоящей работе показана возможность регистрации внутридоплеровских резонансов, обусловленных непосредственно прямой фотоионизацией атомов (или молекул) и особенностями их пролетной релаксации в тонких ячейках с разреженной газовой средой. Такая регистрация может осуществляться по аналогии с методом спектроскопии ультравысокого разре- 
шения, разработанного и апробированного ранее для процессов оптической накачки атомов в подобных ячейках [7,9,12-16]. Структура рассматриваемых внутридоплеровских резонансов существенно зависит от вероятности $\xi$ фотоионизации атомов в единицу времени, а также от отношения $(D / L) \gg 1$ размеров тонкой газовой ячейки (рис. 1). Особый интерес представляет фотоионизационное уширение данных резонансов, которое увеличивается с ростом величины $\xi$ и может быть измерено на эксперименте с высокой точностью известными методами оптической спектроскопии. Для этого необходимо, чтобы подобное фотоионизационное уширение было существенным по сравнению с естественным и остаточным доплеровским уширением исследуемых резонансов. Такое условие возможно при выборе для пробной монохроматической волны резонансного оптического перехода $a \rightarrow b$ (рис. 2) с достаточно малым отношением $\gamma /(k u) \ll 1$ однородной полуширины спектральной линии $\gamma$ к ее доплеровскому уширению $k u$. Кроме того, необходимо использование тонких газовых ячеек с весьма малым отношением размеров $(L / D) \ll 1$ (рис. 1). Например, заданное при расчетах рис. 3-6 отношение $(L / D)=10^{-3}$ имеет место для ячеек с толщиной $L \sim 10 \mu$ m при их поперечном размере $D \sim 1 \mathrm{~cm}$. Такие тонкие ячейки с парами щелочных металлов уже много лет используются в экспериментах $[10,11]$.

Предлагаемый в настоящей работе метод внутридоплеровской спектроскопии позволит получить новую важную информацию о прямой фотоионизации атомов и молекул, которая дополнит результаты уже известных ионизационных методов исследования [1-5].

\section{Список литературы}

[1] Ф.И. Вилесов. УФН, 81 (4), 669 (1963). DOI: $10.3367 / \mathrm{UFNr} .0081 .196312 \mathrm{c} .0669$

[F.I. Vilesov. Sov. Phys. Usp., 6 (6), 888 (1964). DOI: 10.1070/PU1964v006n06ABEH003617].

[2] J.A.R. Samson. Phys. Rep., 28 (4), 303 (1976). DOI: 10.1016/0370-1573(76)90012-0

[3] VUV and Soft X-ray Photoionization. Ed. by U. Becker, D.A. Shirley (Plenum Press, New York and London, 1996).

[4] Н.Б. Делоне, М.В. Федоров. УФН, 158 (2), 215 (1989).

DOI: $10.3367 /$ UFNr.0158.198906b.0215

[N.B. Delone, M.V. Fedorov. Sov. Phys. Usp., $32(6), 500$ (1989). DOI: 10.1070/PU1989v032n06ABEH002725].

[5] W. Demtroder. Laser Spectroscopy: Basic Concepts and Instrumentation. (Springer-Verlag, 1996).

[6] A.Ch. Izmailov. Laser Physics, 2 (5), 762 (1992).

[7] A.Ch. Izmailov. Laser Physics, 3 (2), 507 (1993).

[8] А.Ч. Измайлов. Опт. и спектр., 74 (1), 41 (1993). [A.Ch. Izmailov. Opt. Spectrosc., 74 (1), 25 (1993)].

[9] А.Ч. Измайлов. Опт. и спектр., 75 (3), 664 (1993). [A.Ch. Izmailov. Opt. Spectrosc., 75 (3), 395 (1993)].

[10] S. Briaudeau, D. Bloch, M. Ducloy. Europhys. Lett., 35 (5), 337 (1996). DOI: 10.1209/epl/i1996-00116-1

[11] S. Briaudeau, D. Bloch, M. Ducloy. Phys. Rev. A, 59 (5), 3723 (1999). DOI:10.1103/PhysRevA.59.3723
[12] M. Tachikawa, K. Fukuda, S. Hayashi, T. Kawamura. Jpn. J. Appl. Phys., 37 (12B), L1556 (1998).

[13] M. Otake, K. Fukuda, M. Tachikawa. Appl. Phys. B, 74 (6), 503 (2002).

[14] A.Ch. Izmailov, K. Fukuda, M. Kinoshita, M. Tachikawa. Laser Physics, 14 (1), 30 (2004).

[15] S. Imanishi, U. Tanaka, S. Urabe. Jpn. J. Appl. Phys., 44 (9A), 6767 (2005).

[16] A.Ch. Izmailov. Az. J. Physics: FIZIKA, Section: EN., 17 (1), 3 (2011). (режим доступа в интернете: physics.gov.az/index1.html).

[17] Atomic and molecular processes. Ed. by D.R. Bates (Academic Press. New York and London, 1962). 